\title{
Energy loss of Fe ions in He plasmas at different thermodynamic states
}

Manuel D. Barriga-Carrasco, Luis González-Gallego, Juan Miguel Gil, Rafael Rodríguez, and Guadalupe Espinosa

Citation: Physics of Plasmas 25, 093113 (2018); doi: 10.1063/1.5050528

View online: https://doi.org/10.1063/1.5050528

View Table of Contents: http://aip.scitation.org/toc/php/25/9

Published by the American Institute of Physics

\section{Articles you may be interested in}

Efficient production of strong magnetic fields from ultraintense ultrashort laser pulse with capacitor-coil target Physics of Plasmas 25, 083111 (2018); 10.1063/1.5000991

Influence of gas conditions on parameters of plasma jets generated in the PF-1000U plasma-focus facility

Physics of Plasmas 25, 082715 (2018); 10.1063/1.5045290

Coulomb log for conductivity of dense plasmas

Physics of Plasmas 25, 092707 (2018); 10.1063/1.5053124

Increasing stagnation pressure and thermonuclear performance of inertial confinement fusion capsules by the introduction of a high-Z dopant

Physics of Plasmas 25, 080706 (2018); 10.1063/1.5033459

Conservative algorithms for non-Maxwellian plasma kinetics

Physics of Plasmas 24, 122105 (2017); 10.1063/1.4998242

Slowing down of alpha particles in ICF DT plasmas

Physics of Plasmas 25, 012704 (2018); 10.1063/1.5004213

\section{PHYSICS TODAY}

MANAGER'S GUIDE

WHITEPAPERS

\section{READ NOW}

PRESENTED BY

Accelerate R\&D with Multiphysics Simulation 


\title{
Energy loss of $\mathrm{Fe}$ ions in He plasmas at different thermodynamic states
}

\author{
Manuel D. Barriga-Carrasco, ${ }^{1, a)}$ Luis González-Gallego, ${ }^{1}$ Juan Miguel Gil, ${ }^{2,3}$ \\ Rafael Rodríguez, ${ }^{2,3}$ and Guadalupe Espinosa ${ }^{2}$ \\ ${ }^{1}$ E.T.S.I. Industriales, Universidad de Castilla-La Mancha, E-13071 Ciudad Real, Spain \\ ${ }^{2}$ IUNAT, Departamento de Física, Universidad de Las Palmas de Gran Canaria, E-35017 Las Palmas de Gran \\ Canaria, Spain \\ ${ }^{3}$ Instituto de Fusión Nuclear, Universidad Politécnica de Madrid, E-28006 Madrid, Spain
}

(Received 31 July 2018; accepted 6 September 2018; published online 26 September 2018)

\begin{abstract}
In this work, we analyze the thermodynamic states of the helium plasma and their influence on the stopping power calculations which are needed for obtaining the energy loss of the iron beams traversing them. The analysis is made in ranges of plasma free electron densities $\left(10^{15}-10^{19} \mathrm{~cm}^{-3}\right)$ and temperatures $(1-10 \mathrm{eV})$ of experiments with iron beams at 6 and $4.3 \mathrm{MeV} / \mathrm{u}$ energies. For this purpose, we use Saha-Boltzmann equations for local thermal equilibrium (LTE) and a collisionalradiative model for non-local thermal equilibrium (NLTE) in steady-state situation implemented in a computer code. For the highest temperatures and free electron densities, LTE and NLTE models provide quite similar results for the average ionization and ion abundances. When the opacity effects are taken into account in the NLTE simulations, the optically thick simulations provide fairly similar results to those of the LTE model. The plasma thermodynamic states have a direct impact on the calculation of the energy loss. The differences on the plasma stopping power between considering it in LTE or in NLTE may entail a 10\% of the total stopping for the experiments analyzed in the electron density region of $10^{18}-10^{19} \mathrm{~cm}^{-3}$. These differences can be around $27 \%$ for plasmas with smaller electron density of $10^{17} \mathrm{~cm}^{-3}$ and around $42 \%$ for plasmas with an electron density of $10^{15} \mathrm{~cm}^{-3}$. New experiments would be appreciated to be made in a future to corroborate the latest calculations. Published by AIP Publishing. https://doi.org/10.1063/1.5050528
\end{abstract}

\section{INTRODUCTION}

For the last few decades, the interaction of ion beams and charged particles with plasmas has been an issue widely studied. These interactions have opened a variety of fields in physics to investigate, for example, production and diagnoses of warm dense matter (WDM) ${ }^{1}$ the target response in accelerators, fast ignition, ${ }^{2,3}$ etc. The understanding of the interactions of swift charged particles with plasmas is fundamental to determine the energy deposition of the beam inside the plasma. ${ }^{4-7}$ Therefore, a full theoretical method is needed to calculate correctly this energy deposition of projectile ions in any kind of laboratory plasmas. The energy loss of ions in local thermal equilibrium (LTE) plasmas has been widely studied. However, the energy loss of ions in nonlocal thermal equilibrium (NLTE) plasmas has not been considered so much. The thermodynamic state of the target plasma is so relevant to know its ionization which is, at the same time, crucial to establish the contribution of the plasma free or bound electron to the projectile energy loss. ${ }^{8,9}$

Dielectric formalism can be used to study the free electron contribution based on dielectric functions of the plasma target, ${ }^{10-12}$ for instance, the Random Phase Approximation (RPA) dielectric function. This approximation considers the effect of incident particles as perturbations, so the energy loss is proportional to the square of its charge. RPA dielectric function is interesting as it is valid for plasmas of all degeneracies, ${ }^{13-15}$ although does not consider collisions between

\footnotetext{
a)ManuelD.Barriga@uclm.es
}

target electrons. The Coulomb parameter of the analyzed cases in this work is around $\eta=Q / v_{p}=1.35-1.68$, and then, it is in the limit of the applicability of the RPA, with $Q$ and $v_{p}$ being the charge and velocity of the projectile, respectively.

Mean excitation energies ${ }^{16}$ play an important role in the bound electron stopping power. They have been obtained from Hartree-Fock calculations ${ }^{17,18}$ instead of using complex oscillator strengths sums. ${ }^{8,19}$ Also, it is common to estimate the atomic properties required for the calculation of the excitation energies in the context of the average atom. ${ }^{20}$ However, in this work, they have been obtained in the detailed atom description, where all charge states of the chemical element are considered.

The projectile energy loss depends on plasma thermodynamic state. In general, the laboratory plasmas can be found in LTE or NLTE thermodynamic regimes. In NLTE, ions abundances are calculated by using the so-called collisionalradiative models $(\mathrm{CR})^{21}$ which implies to solve a set of rate equations with coupling of ion configurations, free electrons, and photons. On the other hand, the LTE regime could be reached when the collisional processes are dominant over the radiative ones. The calculation of the ion abundances in LTE is considerable simpler than in NLTE since the SahaBoltzmann (SB) equation can be used. The appropriate knowledge of the thermodynamic regime is a rather important point, since the differences in the ion abundances provided by $\mathrm{CR}$ and $\mathrm{SB}$ simulations can be considerably large. We first analyzed the influence of the thermodynamic regime in the numerical simulation of plasma properties such as the average ionization, ion abundances, and stopping number. For that purpose, we have made NLTE and LTE calculations 
of those properties using the MIXKIP code, ${ }^{22}$ in which both $\mathrm{CR}$ and SB equations are implemented. Furthermore, we have also analyzed the opacity effects in the calculation of the plasma level populations as they can be relevant for the experiments analyzed. In the second part, we have compared our numerical simulations of the energy loss of ion projectiles in plasmas with the data obtained in two experiments.

This paper is organized as follows: In Sec. II, the theoretical models for the calculation of the energy loss and for the thermodynamic states are shown. Afterwards in the same section, the analysis of the influence of the thermodynamic regime in the calculation of plasma properties is presented. Finally, in Sec. III, the results for the energy loss obtained with the theoretical models are discussed and compared with experimental data.

\section{THEORETICAL FRAMEWORK}

\section{A. Ion energy loss}

In this subsection, atomic units were used, $e=\hbar=m_{e}$ $=1$. The energy loss in a partially ionized matter can be estimated through two contributions, free and bound electrons $^{8,23}$

$$
\frac{d E}{d x}=\frac{d E}{d x}_{\text {free }}+\frac{d E}{d x} \text { bound }=\frac{4 \pi Q^{2}}{v_{p}^{2}} n_{a t} \cdot\left(q \cdot L_{f e}+L_{b e}\right) .
$$

The atomic density of the plasma is $n_{a t}$ and the mean plasma ionization is $q$, resulting the free electron density, $n_{f e}=n_{a t} \cdot q$.

The free electron stopping number, $L_{f e}$, can be computed using the dielectric formalism, through RPA dielectric function, $\epsilon_{R P A},{ }^{24,25}$ developed in terms of the wave number $k$ and the frequency $\omega$ provided by quantum mechanics analysis. The expression for RPA dielectric function is ${ }^{10}$

$$
\epsilon_{R P A}(k, \omega)=1+\frac{1}{\pi^{2} k^{2}} \int d^{3} k^{\prime} \frac{f\left(\vec{k}+\overrightarrow{k^{\prime}}\right)-f\left(\overrightarrow{k^{\prime}}\right)}{\omega+i \nu-\left(E_{\vec{k}+\vec{k}^{\prime}}-E_{\overrightarrow{k^{\prime}}}\right)},
$$

where $E_{\overrightarrow{k^{\prime}}}=k^{\prime 2} / 2$, and temperature dependence is shown through the Fermi-Dirac function

$$
f(\vec{k})=\frac{1}{1+\exp \left[\beta\left(E_{k}-\mu\right)\right]},
$$

where $\beta=1 /\left(k_{B} T\right)$ and $\mu$ is the chemical potential of the plasma with an electron density $n_{f e}$ and temperature $T$, and $k_{B}$ is the Boltzmann constant. If the absence of target electron collisions is assumed, the collision frequency $\nu \rightarrow 0$. An analytic RPA dielectric function for plasmas at any degeneracy can be obtained from Eq. $(2)^{24}$

$$
\epsilon_{R P A}(k, \omega)=1+\frac{1}{4 z^{3} \pi k_{F}}[g(u+z)-g(u-z)],
$$

where $u=\omega /\left(k \nu_{F}\right)$ and $z=k /\left(2 k_{F}\right)$ are dimensionless variables $^{10}$ and $k_{F}=\nu_{F}=\sqrt{2 E_{F}}$ is the Fermi velocity. $g(x)$ is defined as

$$
g(x)=\int_{0}^{\infty} \frac{y d y}{\exp \left(D y^{2}-\beta \mu\right)+1} \ln \left(\frac{x+y}{x-y}\right),
$$

where $D=E_{F} \beta$ is the degeneracy parameter. Finally, the free electronic contribution can be calculated as

$$
L_{f e}\left(v_{p}\right)=\frac{1}{2 \pi^{2} n_{f e}} \int_{0}^{\infty} \frac{d k}{k} \int_{0}^{k v_{p}} \omega d \omega \operatorname{Im}\left[-\frac{1}{\epsilon_{R P A}(k, \omega)}\right] .
$$

The bound electron contribution is obtained as the sum of the bound stopping number of each plasma species ${ }^{8,9}$

$$
L_{b e}=\sum_{s}\left(p_{s} \cdot L_{b e, s}\right),
$$

where $p_{s}$ is the relative abundances of the plasma ions, being the bound stopping number of any species $s$

$$
L_{b e, s}=\sum_{i} N_{s, i} L_{b e, s, i}
$$

where $L_{b e, s, i}$ and $N_{s, i}$ are the stopping number and the number of bound electrons in the $i$ shell of the ion species $s$ in the target, respectively. The total bound electron density of the plasma is $n_{b e}=n_{a t} \cdot \sum_{s}\left(p_{s} \cdot N_{s}\right)$, where $N_{s}=\sum_{i} N_{s, i}$.

The stopping number for bound electrons $L_{b e, s, i}$ is obtained from an interpolation between high and low projectile velocity approximations, avoiding the logarithm negative values for low velocities ${ }^{23,25}$

$$
L_{b e, s, i}\left(v_{p}\right)=\left\{\begin{array}{l}
L_{H, i}\left(v_{p}\right)=\ln \left(\frac{2 v_{p}^{2}}{I_{i}}\right)-\frac{2 K_{i}}{v_{p}^{2}} \quad \text { for } v_{p}>v_{\text {int }, i} \\
L_{B, i}\left(v_{p}\right)=\frac{\alpha_{i} v_{p}^{3}}{1+G_{i} v_{p}^{2}} \quad \text { for } v_{p} \leq v_{\text {int }, i},
\end{array}\right.
$$

where $\alpha_{i}=1.067 K_{i}^{1 / 2} I_{i}^{-2}$ is the hydrogenic approximation friction coefficient for low velocities, and $v_{\text {int }, i}=\left(3 K_{i}\right.$ $\left.+1.5 I_{i}\right)^{1 / 2}$ is an intermediate velocity that links both expressions without discontinuity for an electron at the $i$ shell. $G_{i}$ is obtained when $L_{H, i}\left(v_{i n t, i}\right)=L_{B, i}\left(v_{i n t, i}\right)$. The mean excitation energy can be obtained from the following expression: ${ }^{16,26}$

$$
I_{i}=\sqrt{\frac{2 K_{i}}{\left\langle r_{i}^{2}\right\rangle}}
$$

where $K_{i}$ is the electronic kinetic energy and $\left\langle r_{i}^{2}\right\rangle$ is the quadratic mean radius for an electron at the $i$ shell, respectively, using the Hartree-Fock method. ${ }^{17}$ This calculation method has the advantage of estimating the stopping number shell by shell, instead of consider it as a global average value. In Sec. II C, it is shown that how the abundances of the different species are calculated.

\section{B. Ion charge state}

If the ion projectile is considered point-like, the equilibrium charge state, $Q_{\text {eq }}$, can be used in order to estimate the theoretical energy loss. The equilibrium charge state is the charge state that the ion projectile achieves after traveling inside the target till the electron capture and loss processes of the projectile are balanced. It is calculated $\mathrm{as}^{27,28}$ 


$$
Q_{\mathrm{eq}}\left(v_{p}\right)=Z-N_{\mathrm{eq}}\left(v_{p}\right)=Z-Z e^{-v_{r} / Z^{2 / 3}},
$$

where $Z$ is the atomic number of the projectile, $N_{\text {eq }}\left(v_{p}\right)$ is the equilibrium number of bound electrons, $Z^{2 / 3}$ is the velocity of the electrons bound to the projectile in the Thomas Fermi model (a.u.), and $v_{r}$ is the relative velocity of the projectile to the electrons of the target. The equilibrium charge state of the projectile increases together with its relative velocity, unless it achieves the limit value $Q_{\mathrm{eq}}\left(v_{p}=\infty\right)=Z$ when the velocity is high enough.

If the projectile ion is not considered point-like, then the Brandt-Kitagawa (BK) model can be used to describe its charge distribution, using a generic orbital for electrons that depends on the variational parameter $\Lambda$

$$
\rho_{\mathrm{eBK}}(r)=\frac{N}{4 \pi \Lambda^{3}} \frac{\Lambda}{r} e^{-\frac{r}{\Lambda}}
$$

where $N$ is the number of electrons bound to the projectile, and $r$ is the distance to the nucleus. The Fourier transform of the BK electron charge density needed in Eq. (14) is

$$
\rho_{\mathrm{eBK}}(k)=\frac{N}{1+(k \Lambda)^{2}} .
$$

Instead of $N$, we can use the equilibrium number of electrons bound to the projectile obtained just before, $N_{\mathrm{eq}}\left(v_{p}\right)$. Then, the BK distribution can be replaced in the stopping number

$$
\begin{aligned}
L_{f e}\left(v_{p}\right)= & \frac{1}{2 \pi^{2} n_{f e} Q^{2}} \int_{0}^{\infty} \frac{d k}{k}\left[Z-\frac{N_{\mathrm{eq}}\left(v_{p}\right)}{1+\left(k \Lambda\left(v_{p}\right)\right)^{2}}\right]^{2} \\
& \times \int_{0}^{k v_{p}} \omega d \omega \operatorname{Im}\left[-\frac{1}{\epsilon_{R P A}(k, \omega)}\right] .
\end{aligned}
$$

Most authors evaluate the energy loss assuming that the projectile ion does not change its charge state along its travel inside the target. They use the equilibrium charge value; however, it must be considered that although the ion charge tends to it, depending on the target length maybe it will be never achieved. Therefore, the theoretical energy loss calculation should take into account the instantaneous charge state of the projectile during its travel. In our model, the charge state depends on the distance traveled by the projectile inside the target. If $Q_{0}$ is the initial charge state of the ion before entering the target, the charge dependence on the distance is

$$
Q\left(v_{p}, x\right)=Q_{\mathrm{eq}}\left(v_{p}\right)+\left(Q_{0}-Q_{\mathrm{eq}}\left(v_{p}\right)\right) \exp \left(-x / x_{\mathrm{eq}}\right),
$$

where $x_{\text {eq }}$ is the equilibrium length which can be estimated as the distance where the processes of electron capture and loss are equal. ${ }^{29}$ Note that the evolution of the initial charge state Eq. (15) does not depend on the ion velocity, but the equilibrium charge state $Q_{\mathrm{eq}}\left(v_{p}\right)$ does. The parameter $x_{\mathrm{eq}}$ is very important as it determines all the evolution of the charge state and the length that the ion must travel to reach its equilibrium charge state. Then, the number of bound electrons is obtained as $N\left(v_{p}, x\right)=Z-Q\left(v_{p}, x\right)$ and the new free stopping number is

$$
\begin{aligned}
L_{f e}\left(v_{p}, x\right)= & \frac{1}{2 \pi^{2} n_{f e} Q^{2}} \int_{0}^{\infty} \frac{d k}{k}\left[Z-\frac{N\left(v_{p}, x\right)}{1+\left(k \Lambda\left(v_{p}, x\right)\right)^{2}}\right]^{2} \\
& \times \int_{0}^{k v_{p}} \omega d \omega \operatorname{Im}\left[-\frac{1}{\epsilon_{R P A}(k, \omega)}\right]
\end{aligned}
$$

and the one along the propagation in the target plasma is obtained as

$$
L_{f e}\left(v_{p}\right)=\frac{1}{\Delta x} \int_{0}^{\Delta x} L_{f e}\left(v_{p}, x\right) d x
$$

where $\Delta x$ is the total plasma length. The effective charge $Q_{\text {eff }}$ can be defined as the ratio between the energy loss of the heavy ion and that of the proton for the same plasma conditions. $^{27,28}$ This effective charge $Q_{\text {eff }}$ is larger than the equilibrium charge state $Q_{\text {eq }}$ due to the incorporation of the $\mathrm{BK}$ charge distribution

$$
Q_{\mathrm{eff}} \equiv \sqrt{L_{f e}(\mathrm{Fe}) / L_{f e}\left(\mathrm{p}^{+}\right)}
$$

This $Q_{\text {eff }}$ value is used to calculate the energy loss in Sec. III.

\section{Plasma at different thermodynamic states}

When the plasma approaches the LTE regime, the relative abundances of the different ionization stages, $p_{s}$, can be obtained by means of the Saha equation ${ }^{30,31}$

$$
n_{f e} \frac{p_{s+1}}{p_{s}}=\left(\frac{U_{s+1}}{U_{s}}\right)\left(2 \frac{\left(2 \pi k_{B} T\right)^{3 / 2}}{h^{3}}\right) e^{-\frac{\left(\chi_{s}-\Delta y_{s}\right)}{k_{B} T}}
$$

where $U_{s}$ is the partition function of the ion $s, h$ is the Planck constant, $\chi_{s}$ is the ionization potential of the species $s$, and $\Delta \chi_{s}$ is the depression of the ionization potential (continuum lowering, CL) due to the plasma environment. The formulation developed by Stewart and Pyatt ${ }^{32}$ was applied which is valid for the range of densities and temperatures in which ionization is appreciable.

However, for most cases, plasmas do not verify the conditions needed to achieve the LTE regime, so they are in NLTE. The atomic level abundances are then determined from a system of collisional-radiative rate equations. ${ }^{21}$ This set of kinetic rates equations is given by

$$
\frac{d p_{s, i}(\vec{r}, t)}{d t}=\sum_{s^{\prime}, i^{\prime}} p_{s^{\prime}, i^{\prime}}(\vec{r}, t) \mathbb{R}_{s^{\prime}, i^{\prime} \rightarrow s, i}^{+}-\sum_{s^{\prime}, i^{\prime}} p_{s, i}(\vec{r}, t) \mathbb{R}_{s, i \rightarrow s^{\prime}, i^{\prime}}^{-},
$$

where $\vec{r}$ is the position, $t$ is the time, and $p_{s, i}$ is the abundance of the atomic $i$ level of the ion with ionization state $s$ such that $p_{s}=\sum_{i} p_{s, i} . \mathbb{R}_{s^{\prime}, i^{\prime} \rightarrow s, i}^{+}$and $\mathbb{R}_{s, i \rightarrow s^{\prime}, i^{\prime}}^{-}$include all atomic processes that contribute to populate or depopulate, respectively, $s, i$ state. Furthermore, the addition of the abundance of all atomic ions, $p_{s}$, must be equal to the atomic total density and also the plasma must be neutral regarding electric charge. For optically thick plasmas, where the reabsorption of photons plays an important role, these equations are coupled to the radiative transfer equation 


$$
\begin{aligned}
& \frac{1}{c} \frac{\partial A_{I}(\vec{r}, t, \nu, \vec{e})}{\partial t}+\vec{e} \cdot \nabla A_{I}(\vec{r}, t, \nu, \vec{e}) \\
& \quad=-\kappa(\vec{r}, t, \nu) A_{I}(\vec{r}, t, \nu, \vec{e})+j(\vec{r}, t, \nu)
\end{aligned}
$$

where $A_{I}$ is the specific intensity of radiation, $\nu$ is the photon frequency, $\vec{e}$ is an unitary vector in the direction where radiation is propagated, and $\kappa$ and $j$ are the absorption and emission coefficients, respectively, that couple Eqs. (20) and (21). The CR model and the SB equations, used in this work, are implemented in the MIXKIP code. ${ }^{22}$ For optically thick plasmas, assuming stationary conditions for the radiative transfer, the opacity effects are modeled in MIXKIP in an approximate way using the escape factor formalism for the bound-bound opacity ${ }^{33}$ which avoids the explicit resolution of the radiative transfer equation. For a given line transition, $s i \leftrightarrow s j$, the escape factor, $\Lambda_{j i}$, is an alternative way of writing the net rate of line emission leading to an effective reduction in the Einstein spontaneous emission coefficient. We have adopted the technique described in Ref. 34 for the calculation of the escape factors. For a uniform distribution of emitting atoms and isotropic emission in the three basic geometries (plane, cylindrical, and spherical), the escape factor is given by

$$
\Lambda_{j i}=\int_{0}^{\infty} \phi_{i j} \frac{F\left[\tau_{i j}(\nu)\right]}{\tau_{i j}(\nu)} d \nu,
$$

where $\phi_{i j}$ is the line profile. MIKKIP considers a Voigt profile accounting for natural, Doppler, and collisional ${ }^{34}$ broadenings. $\tau_{i j}$ is the optical depth of the line transition and $F\left[\tau_{i j}(\nu)\right]$ is a functional of the optical depth whose particular form depends on the geometry. In this work, we have considered plasmas with cylindrical geometry, and, in this case, the functional is computed by interpolation over a numerically defined function as described in Ref. 34.

Now, it will be shown a concrete analysis of the influence of the atomic kinetics model used to calculate the plasma properties, such as the average ionization and the ion abundances. The ranges of electron temperatures and densities considered are $1-10 \mathrm{eV}$ and $10^{17}-10^{19} \mathrm{~cm}^{-3}$, respectively, which correspond to the plasma conditions obtained in the experiments addressed in this work. ${ }^{35,36}$ This analysis has been performed for a helium plasma in the context of the detailed atomic description, as it is the plasma used for the iron energy loss experiments analyzed in Secs. III A-III C, and the same analysis can be performed for any kind of plasmas, although for low ionized and high-Z plasmas detailed configuration accounting (DCA) description must be used. For the analysis carried out, we have compared the average ionization, the ion abundances, and the stopping number calculated assuming both LTE (SB equations) and NLTE (CR model) in both optically thin and thick approaches, using the MIXKIP code. For the optically thick simulations, we have considered the plasma with cylindrical symmetry with radii of the order of those obtained in the experiments under study, i.e., between 0.25 and $1 \mathrm{~mm}$. In NLTE regime, we have considered the collisional radiative steady state situation (CRSS) since the characteristic time of the evolution of the plasma conditions of the experiments analyzed is around $1 \mu \mathrm{s}$, whereas the characteristic times of the collisional processes are much shorter, around $0.1 \mathrm{~ns}$. The atomic processes included in the CRSS model ${ }^{22}$ were collisional ionization, three body recombination, spontaneous decay, collisional excitation and de-excitation, radiative recombination, autoionization, and electron capture. For non-optically thin NLTE simulations, the spontaneous decay is corrected by the escape factor, as explained before.

Figure 1(a) shows the comparison of the average ionization obtained with the LTE and optically thin NLTE models, as a function of the temperature and for three representative free electron densities of the plasma. We also present a comparison with the non-optically thin NLTE simulations for two plasma radii $(0.25$ and $1 \mathrm{~mm})$ but only for the lowest free electron density, since for the density of $10^{18} \mathrm{~cm}^{-3}$ the results obtained for the average ionization were quite similar to those of the LTE calculation even for the lower radius. As expected, we can observe that the average ionization increases with the temperature and decreases with the free electron density, the latter due to increase in the plasma recombination which is more noticeable at the highest density showed. The differences between models decrease with the increase in free electron density and with the decrease in
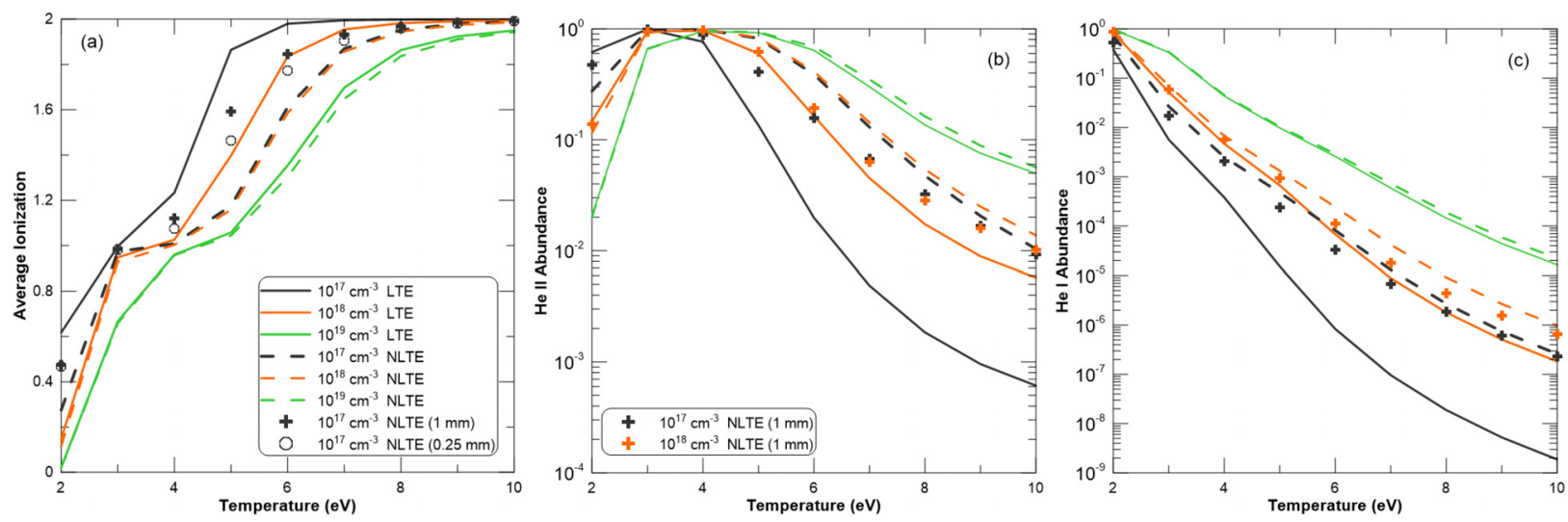

FIG. 1. Comparison of (a) the average ionization, (b) HeII relative abundances, and (c) HeI relative abundances, calculated assuming LTE and NLTE regimes. For the average ionization, non-optically thin NLTE simulations at the free electron density of $10^{17} \mathrm{~cm}^{-3}$ for two plasma radii ( 0.25 and 1 mm) are also shown. For the HeI and HeII relative abundances, the optically thick simulations for a plasma radius of $1 \mathrm{~mm}$ and for free electron densities $10^{17}$ and $10^{18} \mathrm{~cm}^{-3}$ are displayed. 
the temperature, since both factors enhance the relevance of collisional processes and then the LTE regime. The agreement observed for the two lower free electron densities at temperatures higher than $8 \mathrm{eV}$ for NLTE and LTE average ionizations is due to the prevalence of the fully stripped helium ion. We can also detect an agreement for these two densities at the temperature of $3 \mathrm{eV}$ with a prevalence of the HeII ion. A little plateau is observed between 3 and $4 \mathrm{eV}$ associated with the ionization of this ion.

When LTE regime is not achieved, SB model overestimates the average ionization with respect to the NLTE optically thin model, whereas the optically thick simulation always provides results between these two models [see Fig. 1(a)]. At the highest density, LTE and optically thin and thick NLTE results are quite similar in the whole range of temperatures and the plasma could be considered in LTE regime. As the radius of the plasma increases, the opacity effects become more relevant and the results from the optically thick simulations are closer to those of the LTE regime, as the figure shows for the two radii considered. We detect that for the free electron density of $10^{17} \mathrm{~cm}^{-3}$, there are appreciable differences in the average ionization for the optically thick case with respect to both LTE and NLTE optically thin simulations for the electron temperatures range 4-7 eV.

Figure 1(a) also shows that the differences between the average ionizations calculated at different free electron densities in LTE are greater than those in NLTE optically thin case. In LTE regime, the three body recombination is considerable larger than two body processes. The former is more sensitive to the free electron densities than the latter and this could explain the result observed. The fact that the differences between the NLTE average ionizations also increases with the free electron density, for example, between $10^{18}$ and $10^{19} \mathrm{~cm}^{-3}$ (i.e., as the relevance of the three body recombination increases and then NLTE results converge to those in LTE), also endorses this conclusion.

The influence of the atomic kinetics models in the calculation of the abundances of ions HeII and HeI was also analyzed and the results are displayed in Figs. 1(b) and 1(c), respectively. We have shown the optically thick simulations for the free electron densities of $10^{17}$ and $10^{18} \mathrm{~cm}^{-3}$ and the radius of $1 \mathrm{~mm}$, since for the latter density differences in the ion abundances with respect to the other two models are still detected. Figure 1(b) shows that the importance of HeII ion reaches its maximum at temperatures around $3-4 \mathrm{eV}$ and for the highest density this maximum value is retained until almost the temperature of $6 \mathrm{eV}$. Figure 1(c) shows that the abundance of HeI is noticeable only for the two higher densities, due to the increase in the plasma recombination and for temperatures lower than $3 \mathrm{eV}$. For lower densities, its abundance is very small (always lower than $10^{-2}$ ) and is almost negligible for temperatures larger than $2.5 \mathrm{eV}$. For the two lower densities, the abundances of both ions predicted by the LTE simulations decay with the temperature faster than the NLTE simulations, as expected, due to the larger average ionization obtained in the former approach. For the free electron density of $10^{17} \mathrm{~cm}^{-3}$, we can detect noticeable differences between LTE ion abundances and those provided by NLTE model, in both optically thin and thick approaches. These differences decrease with the free electron density and at $10^{19} \mathrm{~cm}^{-3}$ NLTE and LTE results are quite similar, as in the case of the average ionization.

The differences in the average ionization and the ion abundances obtained from the different atomic kinetics models for some ranges of plasma conditions will influence the theoretical calculations of the projectile energy loss in the plasma, Eqs. (6) and (7). To analyze this, we have studied the influence of the atomic kinetics model in the calculation of the total stopping number, $L$

$$
\begin{aligned}
L= & q \cdot L_{f e}+\sum_{s} p_{s} N_{s} L_{b e, s}=q \cdot L_{f e} \\
& +\left(p_{\mathrm{HeI}} N_{\mathrm{HeI}} L_{b e, \mathrm{HeI}}+p_{\mathrm{HeII}} N_{\mathrm{HeII}} L_{b e, \mathrm{HeII}}\right) .
\end{aligned}
$$

Figure 2 shows a comparison for the three free electron densities of the contributions to the stopping number of HeI and HeII ions and of free electrons, as a function of the temperature and obtained with the three atomic kinetics models. The results are closely related to those obtained in the analysis of the ion abundances and the average ionization. Due to the
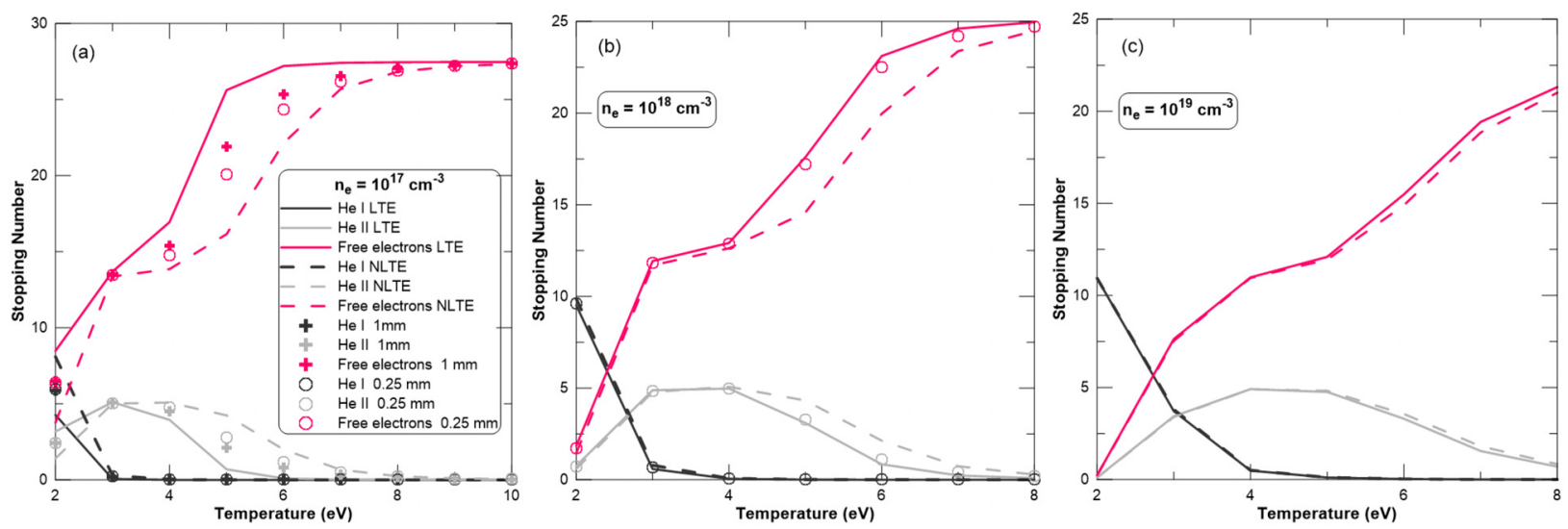

FIG. 2. Comparison of the contributions to the total stopping number, according to Eq. (24), from the stopping number due to free electrons $q L_{f e}$, and the ones due to HeI and HeII ions $\left(p_{s} N_{s} L_{b e, s}\right)$, calculated with different atomic kinetics models, as a function of the electron temperature and for the free electron densities, $n_{e}$ : (a) $10^{17}$, (b) $10^{18}$, and (c) $10^{19} \mathrm{~cm}^{-3}$. For the free electron density, $10^{17} \mathrm{~cm}^{-3}$ NLTE optically thick, 0.25 and $1 \mathrm{~mm}$, simulations are also shown. For $10^{18} \mathrm{~cm}^{-3}$ NLTE optically thick only with $0.25 \mathrm{~mm}$ radii is shown. 
LTE model predicts larger values of the average ionization, the contribution due to the bound electrons is always lower in this case than in NLTE simulations. At the highest density, LTE and NLTE results are quite similar [see Fig. 2(c)]. On the other hand, for the lowest density [see Fig. 2(a)], there are noticeable differences between LTE and NLTE simulations, in both optically thin and thick situations. Figure 2(b) shows that for the free electron density of $10^{18} \mathrm{~cm}^{-3}$, NLTE optically thick simulations provide similar values of the stopping number to those obtained in LTE. The figures also show that the range of temperatures in which the contribution due to bound electrons to the energy loss is not negligible spans as the electron density increases due to the decrease in the average ionization. Thus, for the lowest density, the range is $1-6 \mathrm{eV}$. For the highest density, the range spans until $8 \mathrm{eV}$ and for temperatures up to $5 \mathrm{eV}$ the bound and free contributions are of the same order of magnitude. For the free electron densities of $10^{18}$ and $10^{19} \mathrm{~cm}^{-3}$, we can observe that the bound electron contribution is larger than the free electron one for temperatures lower than 2.5 and $3 \mathrm{eV}$, respectively.

\section{COMPARISONS WITH EXPERIMENTS}

\section{A. Energy loss of Fe ions in He plasmas: Case I}

The experiment of this first case ${ }^{35}$ was performed at the Heavy Ion Medial Accelerator at Chiba (HIMAC) in the National Institute of Radiological Sciences (NIRS), Japan. The experiment measured the energy loss of iron projectiles in a z-pinch discharge plasma. A helium gas is pressurized up to $120 \mathrm{~Pa}$ in a quartz discharge tube, which has an inner diameter of $27 \mathrm{~mm}$ and a length of $160 \mathrm{~mm}$. First, a $2 \mu$ s preionization is done with a peak current of $60 \mathrm{kA}$ by a capacitor that produces a plasma column with a $160 \mathrm{~mm}$ length. Then, the $6 \mathrm{MeV} / \mathrm{u}$ iron projectiles are shot. The ion beam consisted of a number of micro bunches that deliver $2 \times 10^{5}$ ions every $10 \mathrm{~ns}$, which originate from $100 \mathrm{MHz}$ radio frequency acceleration. Figure 3(a) shows the experimental free electron density and temperature time profiles. These were determined using spectrometry assuming the plasma in the LTE regime. The free electron densities ranged from $10^{18}$ to $2.5 \times 10^{18} \mathrm{~cm}^{-3}$ and the electron temperatures from 4.0 to $5.4 \mathrm{eV}$.

In Fig. 3(b), we have represented the average ionizations calculated under LTE and optically thin NLTE assumptions. The figure shows that the differences between both models increase with time, due to the increase in the temperature which enhances the NLTE regime as was commented in Sec. IIC. At $0.95 \mu \mathrm{s}$, the maximum difference is reached, with average ionizations around 1.2 and 1.5 for NLTE and LTE simulations, respectively. The most abundant ions in the plasma are HeII and HeIII with a negligible abundance of HeI ion as Fig. 3(c) shows. For times earlier than $0.8 \mu \mathrm{s}$, both theoretical simulations predict that HeII is the main ion in the plasma. In later times, the relevance of HeIII ion increases, overall in the LTE simulation, and at $1 \mu \mathrm{s}$ the HeII ion abundances provided by the NLTE and LTE simulations have decreased to 0.8 and 0.6 , respectively. The largest abundance of HeII ion in the NLTE calculation implies that the contribution of the bound electrons to the stopping number will be greater than in the LTE simulation, as Fig. 2(b) shows.

This analysis has been made assuming the plasma as optically thin. However, the plasma in the experiment can be assumed to be thick, as an example, a cylinder with a radius of $1 \mathrm{~mm}$. In Sec. II C, we presented NLTE simulations assuming the plasma as optically thick with cylindrical symmetry and with two possible radii, 0.25 and $1 \mathrm{~mm}$. Figure 1(b) shows that for the range of plasma conditions of this experiment, the HeII ion abundances provided by the optically thick simulation were quite similar to those of the LTE simulation. Furthermore, Fig. 2(b) shows that the stopping numbers obtained with the optically thick model agreed with those of the LTE simulation even for the lower plasma radius. Therefore, we can conclude that for the theoretical analysis of this experiment, the plasma can be assumed to be in LTE regime.

For the range of plasma conditions of this experiment, we have compared the values of the average ionization obtained with our LTE model with those provided by Ref. 36 which assumes that the plasma is in LTE as well. The agreement obtained with both models is quite good as Fig. 3(b) reveals. We have also compared our values with those estimated from the SB equations using atomic data from Moore database ${ }^{37}$ (with values of the partition functions, for the three helium ions, $U_{0}=3.46, U_{1}=2.00$, and $U_{2}=1.00$ ) obtaining similar results.

Figures 4(a) and 4(b) show comparisons of experimental and theoretical values of the energy loss of $\mathrm{Fe}^{21+}$ and $\mathrm{Fe}^{25+}$ ions in the helium plasma, respectively, as a function of time. Although we stated before that the plasma can be assumed to be in LTE, we have also represented in the figure the results obtained from the NLTE simulations to illustrate the values that could be obtained in an optically thin plasma.
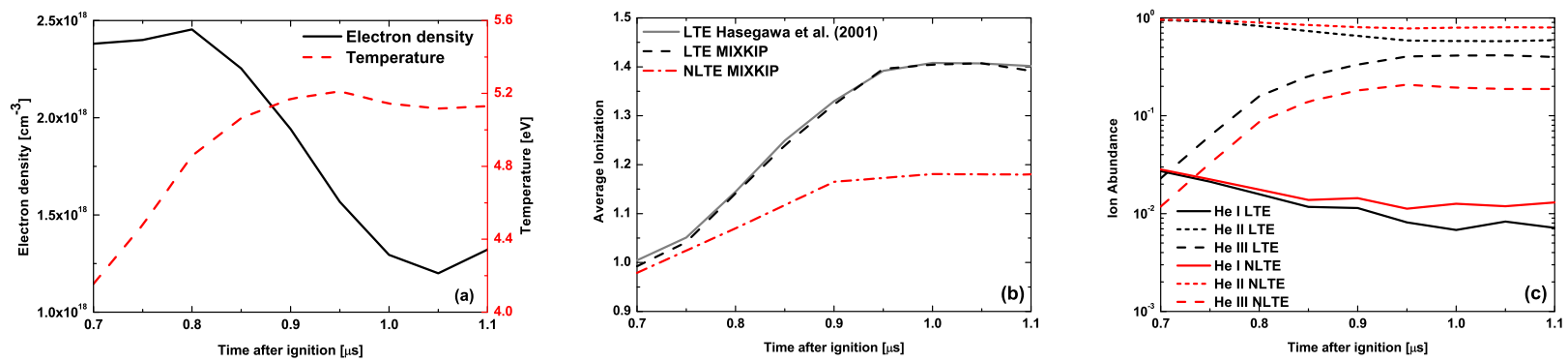

FIG. 3. (a) Free electron temperature and density time profiles of the first experiment. (b) Plasma average ionizations as a function of time calculated with MIXKIP under NLTE optically thin and LTE assumptions and with the LTE model proposed in Ref. 36. (c) Comparison of the abundances of the helium ions as a function of time calculated under NLTE optically thin and LTE assumptions. 

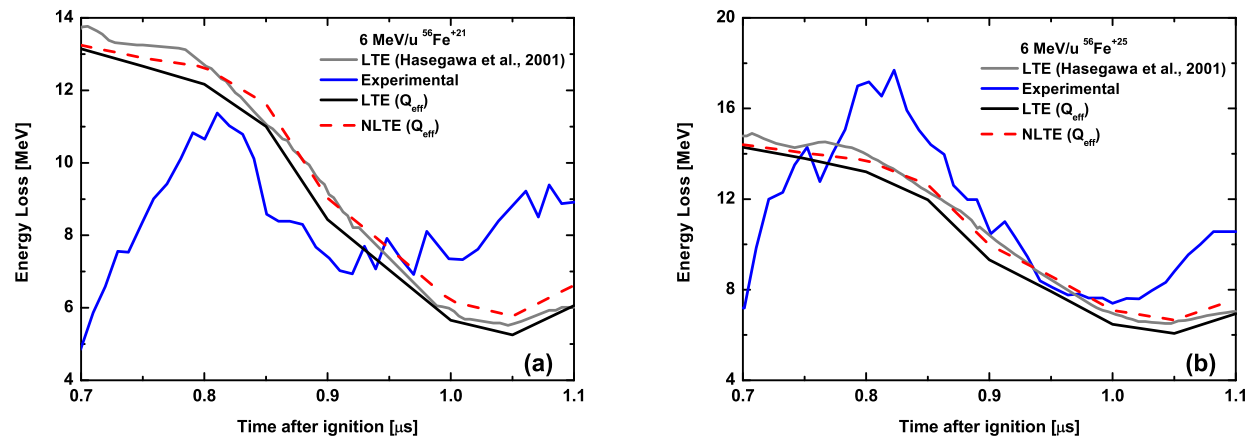

FIG. 4. Energy loss of $6 \mathrm{MeV} / \mathrm{u}$ projectiles of (a) $\mathrm{Fe}^{21+}$ and (b) $\mathrm{Fe}^{25+}$ ions as function of plasma evolution time. Calculations (Grey solid line) and experimental data (Blue solid line) from Hasegawa et al. $^{35}$ Our calculations: LTE (Black line) and NLTE (Red dotted line).
We can observe that the energy loss predicted by the optically thin calculations is slightly greater than those obtained from the LTE simulation. This fact implies that the lower free electron contribution to the stopping number in the optically thin situation [see Fig. 2(b)] is offset by the bound electron contribution. Both NLTE and LTE curves of the energy losses have a similar behavior, decreasing in time for almost the whole range, and they are very similar to the free electron density pattern shown in Fig. 3(a) as the energy loss is almost proportional to the density. Figures 4(a) and 4(b) show that the energy loss is larger as the charge of the projectile increases as is expected according to Eqs. (6) and (8) for the free and bound electron contributions, respectively. The agreement between the theoretical simulations and the experimental data is better for beams of $\mathrm{Fe}^{25+}$ ions than for $\mathrm{Fe}^{21+}$. Furthermore, the agreement is also better for times later than $0.8 \mu \mathrm{s}$. This could be due to two factors: first, the errors in the free electron density obtained from the Stark broadening of a HeI line are greater at the earliest times; ${ }^{35}$ second, the error bars in the experimental energy loss are also greater for times between 0.70 and $0.75 \mu \mathrm{s}$.

We have also presented in Figs. 4(a) and 4(b) the theoretical values for the energy loss obtained in Hasegawa et al. $^{35}$ In that work, the energy loss is obtained from the following equation:

$$
-\frac{d E}{d x}=\frac{4 \pi Q^{2}}{v_{p}^{2}} n_{a t}\left[q L_{f e}+P \ln \left(\frac{2 \gamma v_{p}^{2}}{\bar{I}}\right)\right],
$$

where $P=\sum_{s}\left(p_{s} \cdot N_{s}\right)$ is supposed to be the averaged electronic population for an average atom in the plasma and $\gamma=\left(1-v_{p}^{2} / c^{2}\right)^{-1 / 2}$ is the Lorentz factor, which is approximately one for the projectile energies. The stopping number for free electrons, $L_{f e}$, is defined with the following expression:

$$
L_{f e}=\min \left\{\ln \left(\frac{\gamma v_{p}^{3}}{Q \omega_{p}}\right), \ln \left(\frac{2 \gamma v_{p}^{2}}{\omega_{p}}\right)\right\},
$$

where $\omega_{p}=\left(4 \pi n_{a t} q\right)^{1 / 2}$ is the plasma frequency, assuming the plasma to be in LTE. The figures show small differences between the energy losses obtained with both LTE models. Hence, the ratio of the observed energy losses of $\mathrm{Fe}^{25+}$ to $\mathrm{Fe}^{21+}$ ions was about 1.6, whereas the ratio obtained from the theoretical model used in Ref. 36 was around 1.1 and the ratio provided by our model is 1.12 . The first thing we realize is that the experimental energy ratio cannot be possible, because the ratio between the charge of the two ions has to diminish at the same time the ions deepen in the plasma approaching to equilibrium value, so this ratio must be always less than the initial one, $(25 / 21)^{2}=1.42$. In our model, the effective charge is used Eq. (18) where, besides the BK charge distribution, the equilibrium charge state is also included. Our calculated energy loss is nearly the same for the $\mathrm{Fe}^{25+}$ to $\mathrm{Fe}^{21+}$ ions; it means that both ions tend rapidly to the equilibrium charge state although this charge state is not reached at the end of their travel in this kind of low density plasmas. The effect of the BK charge distribution is always to increase the value of the effective charge. Both theoretical models, Hasegawa and ours, slightly differ in the energy loss because of how it was calculated: They use Bethe logarithm for high projectile velocities for free and bound electron stopping number Eq. (24), while we use the dielectric formalism for free electrons and an interpolation between a more exact adjustment for high and low velocities in the case of bound electrons. Furthermore, they use an average atom model for the calculation of the mean excitation energies, whereas in this work a detailed description is employed.

\section{B. Energy loss of Fe ions in He plasmas: Case II}

The second work ${ }^{36}$ was conducted on the same facility as the previous one. The quartz tube used had an inner diameter of $30 \mathrm{~mm}$ and contains a $300 \mathrm{~Pa}$ helium gas. The gas was preionized with a discharge that reached $70 \mathrm{kA}$, and a plasma column of $160 \mathrm{~mm}$ length was produced $1 \mu \mathrm{s}$ after the discharge ignition. The 4.3 and $6 \mathrm{MeV} / \mathrm{u}$ iron projectiles were shot afterwards. The authors carried out numerical analysis about the plasma state using a magneto-hydrodynamic code. Spectroscopy and hydrodynamic simulations showed that the plasma was uniform around the z-pinch setup axis for a $2 \mathrm{~mm}$ diameter. Figure 5(a) shows the free electron density and temperature time profiles, which were obtained from the spectroscopic analysis assuming the plasma to be in LTE. Free electron density ranged from $2.0 \times 10^{18}$ to $1.4 \times 10^{19} \mathrm{~cm}^{-3}$ and the temperature from 4.5 to $6.5 \mathrm{eV}$.

According to the analysis presented at the end of Sec. II C for the lower free electron densities of the experiment range, around $2.0 \times 10^{18}$, there are some differences between NLTE and LTE results for the average ionization and the ion abundances, as Figs. 5(b) and 5(c) reveal. For example, it can be observed that the maximum differences in the average ionization are around 5\%. However, as in the previous experiment, the helium plasma has a radius of $1 \mathrm{~mm}$, and therefore, opacity effects should be included in the NLTE simulations. 

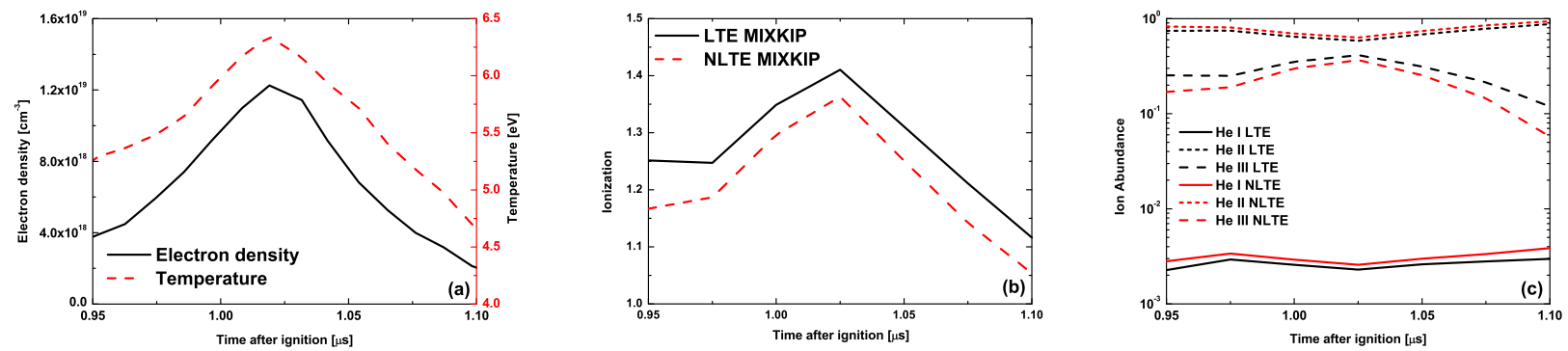

FIG. 5. (a) Electron temperature and density time profiles of the second experiment. (b) Plasma average ionizations as a function of time calculated with MIXKIP under LTE and NLTE assumptions. (c) Comparison of the abundances of helium ions as a function of time calculated under LTE and NLTE assumptions.

In that case, the results provided by the LTE and NLTE models are quite similar for both the average ionization and the ion abundances and also for the energy loss as concluded in the analysis of the previous experiment. For the range of higher densities of this experiment, around $1.4 \times 10^{19} \mathrm{~cm}^{-3}$, the differences between the optically thin NLTE and the LTE simulations are lower and when the opacity effects are taken into account the agreement obtained between the nonoptically thin NLTE and the LTE calculations is even better than in the range of lower free electron densities. Therefore, the assumption in this experiment of LTE regime for the plasma is appropriate.

In this experiment, the free electron densities reached are higher than in the previous one. The increase in the density leads to a larger recombination and lower average ionizations, as Fig. 1 shows. This fact implies that the relevance of the bound electron contribution to the energy loss will be strengthened with respect to the previous experiment. From the time profiles, we can observe that, in general, the ranges of lower $\left(2 \times 10^{18} \mathrm{~cm}^{-3}\right)$ and higher free electron densities $\left(1 \times 10^{19} \mathrm{~cm}^{-3}\right)$ coincide with the ranges of lower $(4.5-5.2 \mathrm{eV})$ and higher temperatures $(5.5-6.5 \mathrm{eV})$. This fact makes that the bound and free electron contributions to the stopping number have values, between 5-3 and 12-15, respectively [see Figs. 2(b) and 2(c)], for the range of plasma conditions of the experiment. Therefore, the relative contribution, in percentage, of the bound electron to the stopping number in this experiment is between $42 \%$ and $20 \%$, whereas for the previous experiment the range was $40 \%-10 \%$.

In Fig. 6, we have represented the comparison of the experimental data with our simulations performed in LTE and in NLTE optically thin approach, calculated with the effective charge state of the projectiles Eq. (18). The initial charge states in each experiment were $\mathrm{Fe}^{21+}$ and $\mathrm{Fe}^{23+}$ for $6 \mathrm{MeV} / \mathrm{u}$ energy and $\mathrm{Fe}^{20+}$ and $\mathrm{Fe}^{22+}$ for $4.3 \mathrm{MeV} / \mathrm{u}$ energy. We observe that the NLTE and LTE simulations provide quite similar results. We have obtained that the NLTE optically thick calculations present an excellent agreement with the LTE ones, as expected according to the analysis presented in Sec. IIC. In this case II, there is not a theoretical curve provided by the experimentalists to compare with. As in the previous experiment, all the curves of the energy loss reproduce the pattern of the time profile of the free electron density. The figures show that the energy loss is higher for slower projectiles, what is expected according to Eqs. (6) and (8). We can observe that, in general, calculations carried out with different initial charge states but with the same
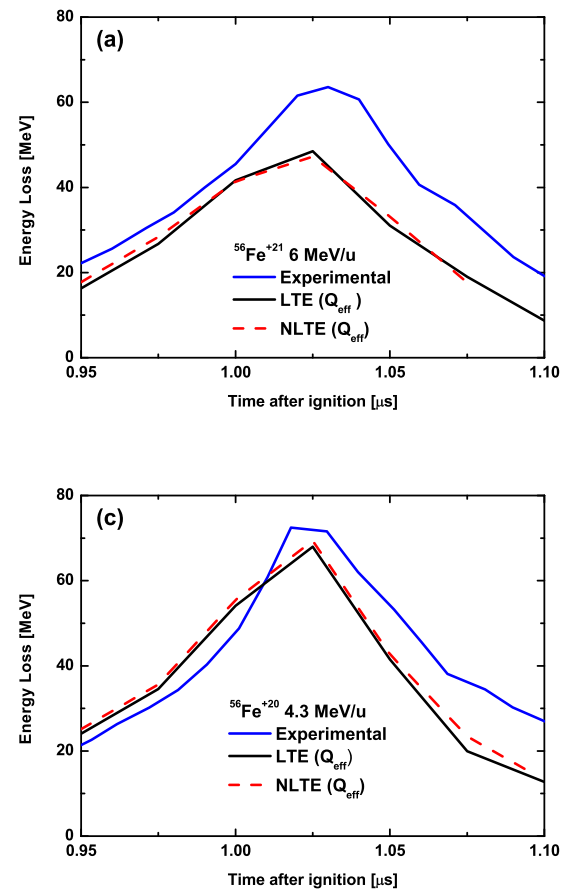
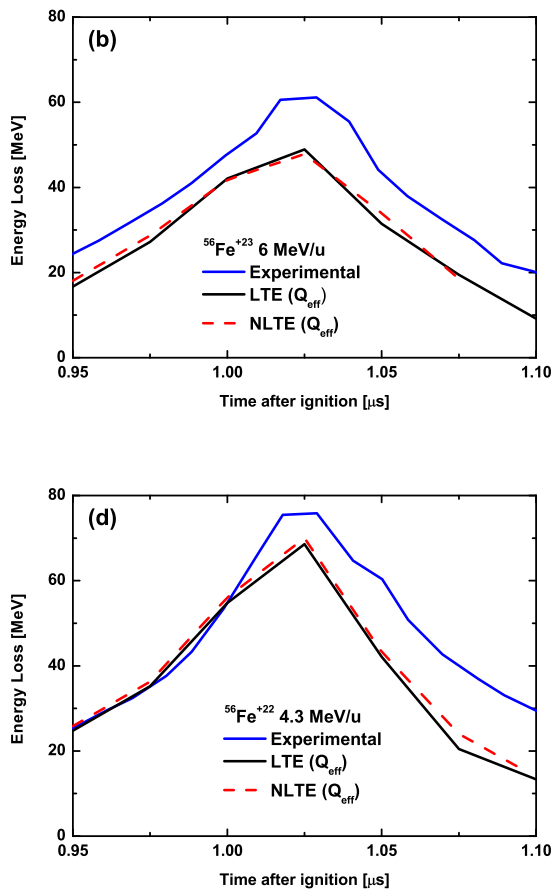

FIG. 6. Energy loss of $6 \mathrm{MeV} / \mathrm{u} \mathrm{Fe} \mathrm{Fe}^{21+}$ $\mathrm{Fe}^{23+}$ ions and $4.3 \mathrm{MeV} / \mathrm{u} \mathrm{Fe}^{20+}, \mathrm{Fe}^{22+}$ ions as function of plasma evolution time. Experimental data from Hasegawa et $a .^{36}$ (Blue solid line). Our calculations with initial projectile charge state: LTE (Black line) and NLTE (Red dotted line). 
velocity show a similar energy loss. This means that the projectiles reach their equilibrium charge state very soon inside this kind of plasma which is more dense than in the case I; this equilibrium charge state is the same for all iron ions with the same energy, Eq. (11). We can also realize that our simulations fit better to the lower energy ions than for higher energy ions. This is because our calculated charge state at higher energies is smaller than the real value, meaning that actually the capture of electrons must be lower than it is considered in our model.

\section{Energy loss of Fe ions in He plasmas: Optimal case}

Once analyzed the previous cases, Secs. III A and III B, it is seen that for plasma densities used in these experiments the differences in energy loss are very small between considering the plasma in LTE or NLTE states, see Fig. 2(b). Obviously, this is because at these plasma densities, the plasma would not have such a large different ionization considering it was at LTE or NLTE, see Fig. 1(a). On the other hand, it is observed that the temperatures of the two experiments are in the zone of greater ionization difference between considering the plasma in the one or in the other state. Therefore, in this section, the temperature will be constant at $5 \mathrm{eV}$ and the ionization and the stopping power of the plasma will be analyzed in more detail according to its density and its thermodynamic state. This would help us to establish what the most ideal plasma conditions to distinguish in what thermodynamic state the plasma is, by the energy loss of ion projectiles.

Figure 7(a) presents the plasma ionization as a function of its total electron density at $5 \mathrm{eV}$ considering the plasma to be in LTE or NLTE. It is easily seen that the difference in ionization according to the plasma state increases with decreasing density. It must be borne in mind that for very low plasma densities it can no longer be considered in LTE and must be considered in the corona regime (corona equilibrium, CE). In any case, for a temperature of $5 \mathrm{eV}$, it is analyzed that for the whole range of densities studied here, it is valid to differentiate between the plasma in LTE and NLTE.

Figure 7(b) shows the relative stopping between the case that the plasma is considered in LTE or in NLTE as a function of the total electron density of the plasma at a temperature of $5 \mathrm{eV}$. It can also be seen the contribution to the total stopping of the bound and free electrons. In all three cases, the relative differences in absolute values become greater at smaller densities. The stopping of free electrons in the case of considering the plasma in LTE state is always greater than the stopping of free electrons in the case of considering the plasma in the NLTE state, as would be expected since the stopping of free electrons is proportional to the ionization. On the other hand, the stopping of bound electrons in LTE is always lower than in NLTE, since the stopping of bound electrons is contrary to ionization. All this means that the total stopping is always higher in the LTE state than in the NLTE, but not as much as in the case of considering only the stopping of free electrons. The greater difference for free electrons is reduced by the bound electron contribution.

Finally, as it has been seen that for an approximate density of $n_{e}=1 \times 10^{17} \mathrm{~cm}^{-3}$ and a temperature of $5 \mathrm{eV}$, the difference between the total stopping of the plasma between considering it in LTE or in NLTE is sufficiently large; it will be analyzed for this case the dependence of the total stopping with the incident energy of the ion projectile. Figure 7(c) shows how the relative difference between the two stoppings increases very slightly as the projectile energy decreases. The relative values goes from $27 \%$ at the highest energies, around $6 \mathrm{MeV} / \mathrm{u}$ as in the experiments in Secs. III A and III B, to $32 \%$ at the smallest energies studied. This indicates that the relative stopping has little dependence on the projectile, since it does not depend so much on its incident energy and not also on its charge state, since the relative stopping does not depend on the projectile's charge.

\section{CONCLUSIONS}

In this work, we have first carried out an analysis of the thermodynamic states (LTE and NLTE) of the helium plasma which are needed for obtaining the energy loss of ion beams traversing them. The analysis was made in ranges of free electron densities $\left(10^{17}-10^{19} \mathrm{~cm}^{-3}\right)$ and temperatures $(1-10 \mathrm{eV})$ of experiments with iron beams. ${ }^{35,36}$ For this purpose, we have made comparisons of those properties calculated with Saha-Boltzmann equations and a collisionalradiative model in steady-state situation implemented in the MIXKIP code, in order to reproduce LTE and NLTE regimes, respectively. Since the opacity effects influence in the calculation of the plasma level populations, NLTE optically thick numerical simulations were performed assuming the plasma with cylindrical symmetry with two possible radii, 0.25 and $1 \mathrm{~mm}$. In the CRSS model, the photon selfabsorption was modeled through the escape factor formalism. For the free electron density of $10^{19} \mathrm{~cm}^{-3}$, LTE and
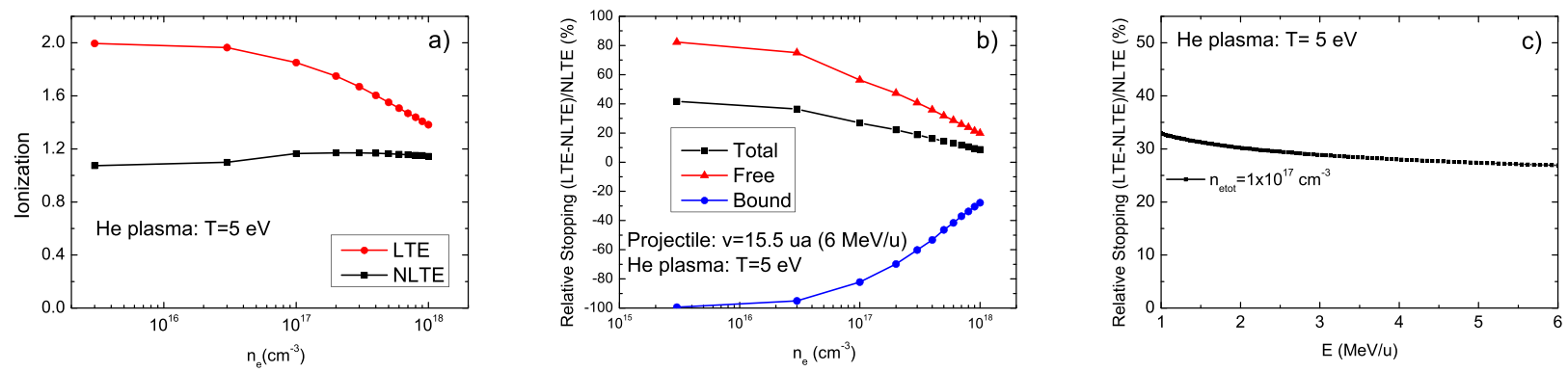

FIG. 7. (a) Plasma ionization as a function of its total electron density considering the plasma in LTE or NLTE. (b) Relative stopping between the plasma considered in LTE or in NLTE as a function of the total electron density. (c) Difference between the total stopping of the plasma in LTE or in NLTE as a function of the incident projectile energy. 
NLTE models provide quite similar results for the average ionization and ion abundances in the whole range of plasma temperatures. On the other hand, noticeable differences are obtained for the lowest density although for the average ionization for temperatures larger than $8 \mathrm{eV}$ both simulations agree but due to the prevalence of the fully stripped ion. For the free electron density of $10^{18} \mathrm{~cm}^{-3}$, similar results are obtained for temperatures up to about $4 \mathrm{eV}$. When the opacity effects are taken into account in the NLTE simulations, it is obtained that for the density of $10^{18} \mathrm{~cm}^{-3}$ the optically thick simulations provide fairly similar results to those of the LTE model. On the other hand, for the lowest density considered, although the optically thick results are closer to the LTE ones than in the optically thin case, differences between both models are still detected.

The theoretical model used in this work to calculate the energy loss is based on the dielectric formalism, through a RPA dielectric function, for the contribution of the free electrons and on an interpolation between high and low projectile velocity approximations for the bound electron contribution. We have obtained that the relevance of this last contribution increases with the density due to the increase in plasma recombination and the resulting decrease in the average ionization. We have obtained that this contribution may entail a $40 \%$ of the total stopping number for ranges of plasma conditions reached in the experiments analyzed in this work. We have also obtained that the abundance of HeI ion is only relevant for temperatures lower than $4 \mathrm{eV}$. Since in the experiments analyzed the temperatures achieved are always greater, the bound electron contribution to the stopping number will be due mainly to HeII ion. The influence of the atomic kinetics models in the average ionization and ion abundances has a direct impact on the calculation of this property and, for this reason, the same conclusions before commented were obtained for the stopping number. Since the free electron densities of the two experiments analyzed are between $10^{18}$ and $10^{19} \mathrm{~cm}^{-3}$, the plasma can be assumed to be in LTE.

We have used these results to address the theoretical analysis of two experiments performed for the purpose of studying the energy loss of iron projectiles with different charges in helium plasmas. Both experiments were conducted on the same facility where z-pinch discharge helium plasmas were obtained, with a length of $160 \mathrm{~mm}$ and radius of $1 \mathrm{~mm}$, and, then, iron projectiles were shot. The free electron densities reached in the case II were higher than in the case I. In any case, we obtained in both experiments that the NLTE optically thick simulations provide quite similar results to those obtained in LTE calculations and, therefore, the helium plasmas could considered in LTE regime. In general, our theoretical calculations showed a good agreement for the energy loss with the experimental values, although the agreement was better for the second experiment analyzed, especially a lower energies. In the first experiment, the authors also provided a theoretical simulation of the energy loss, but the agreement with the experimental results was not so fair, but it is similar than our simulation. The simulations are less sensitive to the change of the projectile charge than those provided by the experimental data, which means that the simulations consider both ions tend rapidly to their equilibrium charge state although this state is not reached at the end of their travel in this kind of low density plasmas. In the second experiment, we have shown that the experimental and our calculations on the energy loss are just a little sensitive to the initial value of the projectile charge. This means that the projectiles reach their equilibrium charge in the plasma range as in the second experiment the plasma density is higher.

Finally, a more detailed study has been made to analyze the dependence of the projectile energy loss, or the plasma stopping, in LTE or in NLTE with the density of the plasma. It has been seen that the difference in energy loss between the two thermodynamic cases of the plasma is significantly larger as the plasma density is lower, but it would be much larger if we did not consider the energy loss with the plasma bound electrons. At the same time, it has been seen that the difference in the energy loss of the projectile according to the plasma state depends very lightly on the characteristics of the projectile, that is, to say, on its energy and its charge.

\section{ACKNOWLEDGMENTS}

L.G.-G. is grateful to Vicerrectorado de Investigación y Política Científica de la UCLM for the APPLe Collaboration Grant. Also, this work has been supported by the EUROfusion Consortium TASK AGREEMENT WPENR: Enabling Research IFE, Project No. AWP15-ENR-01/CEA02 and by the Project of the Spanish Government with Reference No. FIS2016-81019-P.

${ }^{1}$ D. Casas, A. A. Andreev, M. Schnrer, M. D. Barriga-Carrasco, R Morales, and L. Gonzalez-Gallego, Laser Part. Beams 34, 306 (2016).

${ }^{2}$ M. Roth, T. Cowan, M. Key, S. Hatchett, C. Brown, W. Fountain, J. Johnson, D. Pennington, R. Snavely, S. Wilks et al., Phys. Rev. Lett. 86, 436 (2001).

${ }^{3}$ M. Tabak, J. Hammer, M. E. Glinsky, W. L. Kruer, S. C. Wilks, J. Woodworth, E. M. Campbell, M. D. Perry, and R. J. Mason, Phys. Plasmas 1, 1626 (1994).

${ }^{4}$ V. Mintsev, V. Gryaznov, M. Kulish, A. Filimonov, V. Fortov, B. Sharkov, A. Golubev, A. Fertman, V. Turtikov, A. Vishnevskiy et al., Contrib. Plasma Phys. 39, 45 (1999).

${ }^{5}$ C. Deutsch, N. Tahir, M. Barriga-Carrasco, V. Ceban, P. Fromy, D. Gilles, D. Leger, G. Maynard, B. Tashev, and L. Volpe, Nucl. Instrum. Methods Phys. Res., Sect. A 733, 39 (2014).

${ }^{6}$ A. Zylstra, J. Frenje, P. Grabowski, C. Li, G. Collins, P. Fitzsimmons, S. Glenzer, F. Graziani, S. Hansen, S. Hu et al., Phys. Rev. Lett. 114, 215002 (2015).

${ }^{7}$ G. Xu, M. D. Barriga-Carrasco, A. Blazevic, B. Borovkov, D. Casas, K. Cistakov, R. Gavrilin, M. Iberler, J. Jacoby, G. Loisch, R. Morales, R. Mäder, S.-X. Qin, T. Rienecker, O. Rosmej, S. Savin, A. Schönlein, K. Weyrich, J. Wiechula, J. Wieser, G. Q. Xiao, and Y. T. Zhao, Phys. Rev. Lett. 119, 204801 (2017).

${ }^{8}$ D. Casas, M. D. Barriga-Carrasco, and J. Rubio, Phys. Rev. E 88, 033102 (2013).

${ }^{9}$ M. D. Barriga-Carrasco and D. Casas, Laser Part. Beams 31, 105 (2013).

${ }^{10}$ J. Lindhard, Kgl. Danske Videnskab. Selskab Mat.-Fys. Medd. 28, 1 (1954).

${ }^{11}$ N. D. Mermin, Phys. Rev. B 1, 2362 (1970).

${ }^{12}$ T. Peter and J. Meyer-ter Vehn, Phys. Rev. A 43, 1998 (1991).

${ }^{13}$ M. D. Barriga-Carrasco, Phys. Rev. E 82, 046403 (2010).

${ }^{14}$ M. D. Barriga-Carrasco, Laser Part. Beams 28, 307 (2010).

${ }^{15}$ M. D. Barriga-Carrasco, Laser Part. Beams 29, 81-86 (2011).

${ }^{16}$ X. Garbet, C. Deutsch, and G. Maynard, J. Appl. Phys. 61, 907 (1987).

${ }^{17}$ C. F. Fischer, Comput. Phys. Commun. 43, 355 (1987). 
${ }^{18}$ H. Haken, W. D. Brewer, and H. C. Wolf, The Physics of Atoms and Quanta: Introduction to Experiments and Theory, Advanced Texts in Physics (Springer, 2006).

${ }^{19}$ R. Bell, D. Bish, and P. Gill, J. Phys. B: At., Mol. Opt. Phys. 5, 476 (1972).

${ }^{20} \mathrm{H}$. Mayer, Methods of opacity calculations. Report LA-647, Los Alamos Scientific Laboratory, Los Alamos, NM, 1947.

${ }^{21}$ R. McWhirter, Phys. Rep. 37, 165 (1978).

${ }^{22}$ G. Espinosa, R. Rodríguez, J. M. Gil, F. Suzuki-Vidal, S. V. Lebedev, A. Ciardi, J. G. Rubiano, and P. Martel, Phys. Rev. E 95, 033201 (2017).

${ }^{23}$ M. D. Barriga-Carrasco, G. Maynard, and Y. K. Kurilenkov, Phys. Rev. E 70, 066407 (2004).

${ }^{24}$ N. R. Arista and W. Brandt, Phys. Rev. A 29, 1471 (1984).

${ }^{25}$ G. Maynard and C. Deutsch, J. Phys. (Paris) 46, 1113 (1985).

${ }^{26}$ M. D. Barriga-Carrasco and G. Maynard, Laser Part. Beams 23, 211 (2005).

${ }^{27}$ M. D. Barriga-Carrasco, Phys. Rev. E 88, 043107 (2013).
${ }^{28}$ M. D. Barriga-Carrasco, D. Casas, and R. Morales, Phys. Rev. E 93, 033204 (2016).

${ }^{29}$ R. Morales, M. D. Barriga-Carrasco, and D. Casas, Phys. Plasmas 24, 042703 (2017).

${ }^{30}$ M. N. Saha, Proc. R. Soc. London, Ser. A 99, 135 (1921).

${ }^{31}$ R. O. Dendy, Plasma Physics: An Introductory Course (Cambridge University Press, 1995), p. 476.

${ }^{32}$ J. Stewart and K. Pyatt, Astrophys. J. 144, 1203 (1966).

${ }^{33}$ R. Mancini, R. Joyce, and C. Hooper, Jr., J. Phys. B: At. Mol. Phys. 20, 2975 (1987).

${ }^{34}$ M. S. Dimitrijevic and N. Konjevic, Astron. Astrophys. 172, 345 (1987).

${ }^{35}$ J. Hasegawa, Y. Nakajima, K. Sakai, M. Yoshida, S. Fukata, K. Nishigori, M. Kojima, Y. Oguri, M. Nakajima, K. Horioka et al., Nucl. Instrum. Methods Phys. Res., Sect. A 464, 237 (2001).

${ }^{36}$ J. Hasegawa, N. Yokoya, Y. Kobayashi, M. Yoshida, M. Kojima, T. Sasaki, H. Fukuda, M. Ogawa, Y. Oguri, and T. Murakami, Laser Part. Beams 21, 7 (2003).

${ }^{37}$ C. E. Moore, US National Bureau of Standards Circular (1949), p. 467. 